\title{
Cluster randomized evaluation of Adolescent Girls Empowerment Programme (AGEP): study protocol
}

\author{
Paul C. Hewett ${ }^{1 *}$, Karen Austrian², Erica Soler-Hampejsek ${ }^{3,4}$, Jere R. Behrman ${ }^{5}$, Fiammetta Bozzani ${ }^{6}$
} and Natalie A. Jackson-Hachonda ${ }^{7}$

\begin{abstract}
Background: Adolescents in less developed countries such as Zambia often face multi-faceted challenges for achieving successful transitions through adolescence to early adulthood. The literature has noted the need to introduce interventions during this period, particularly for adolescent girls, with the perspective that such investments have significant economic, social and health returns to society. The Adolescent Girls Empowerment Programme (AGEP) was an intervention designed as a catalyst for change for adolescent girls through themselves, to their family and community.
\end{abstract}

Methods/design: AGEP was a multi-sectoral intervention targeting over 10,000 vulnerable adolescent girls ages 10-19 in rural and urban areas, in four of the ten provinces of Zambia. At the core of AGEP were mentor-led, weekly girls' group meetings of 20 to 30 adolescent girls participating over two years. Three curricula - sexual and reproductive health and lifeskills, financial literacy, and nutrition — guided the meetings. An engaging and participatory pedagogical approach was used. Two additional program components, a health voucher and a bank account, were offered to some girls to provide direct mechanisms to improve access to health and financial services. Embedded within AGEP was a rigorous multi-arm randomised cluster trial with randomization to different combinations of programme arms. The study was powered to assess the impact across a set of key longer-term outcomes, including early marriage and first birth, contraceptive use, educational attainment and acquisition of HIV and HSV-2. Baseline behavioural surveys and biological specimen collection were initiated in 2013. Impact was evaluated immediately after the program ended in 2015 and will be evaluated again after two additional years of follow-up in 2017. The primary analysis is intent-to-treat. Qualitative data are being collected in 2013, 2015 and 2017 to inform the programme implementation and the quantitative findings. An economic evaluation will evaluate the incremental costeffectiveness of each component of the intervention.

Discussion: The AGEP program and embedded evaluation will provide detailed information regarding interventions for adolescent girls in developing country settings. It will provide a rich information and data source on adolescent girls and its related findings will inform policy-makers, health professionals, donors and other stakeholders.

Trial registration: ISRCTN29322231. March 04 2016; retrospectively registered.

Keywords: Adolescent girls, Randomized trial, Multi-sectoral, Zambia, Empowerment, Savings, Financial education, Nutrition education, Sexual and reproductive health, HIV, HSV-2

\footnotetext{
* Correspondence: phewett@popcouncil.org

'Population Council, 4301 Connecticut Avenue, Washington, D.C. 2008, USA

Full list of author information is available at the end of the article
} 


\section{Background}

This paper reviews the design and methods of a multiarm, randomized cluster evaluation of the Adolescent Girls Empowerment Programme (AGEP) in Zambia. The programme was designed as a multi-sectoral intervention targeted to vulnerable, rural and urban adolescent girls ages 10-19. Embedded in the design of the programme was a rigorous, randomized evaluation that recruited a cohort of eligible adolescent girls invited to participate in the programme and girls residing in control areas. The evaluation sample was interviewed at baseline in late 2013 and early 2014 and is being tracked annually through 2017, with mid-term results collected in 2015 immediately after the end of the intervention and endline results in 2017, two years post programme termination [1].

Adolescent girls in less developed countries face a variety of risks and challenges in achieving positive and successful transitions to adulthood [2]. In Zambia, a significant proportion of girls enter marriage and/or begin childbearing early, even prior to the expected age of school leaving. As indicated by the 2013-2014 Zambia Demographic and Health Survey (DHS), nearly one in three girls aged 20-24 had married by age 18, with a similar percentage having begun childbearing [1]. The prevalence in rural areas is nearly double that of urban areas for both early marriage (39\% versus $22 \%$ ) and early childbearing (42\% versus $21 \%$ ) [3]. Not surprisingly, given rates of early marriage and childbearing, the risk of school leaving for girls during the school age years is high, with $18 \%$ of primary school age girls and $59 \%$ of secondary age girls not currently attending school [1]. The Zambia DHS also indicates that girls in Zambia begin sexual activity early, have low rates of condom use and have risk behaviours that increase their chances of acquiring sexually transmitted infections (STIs), including HIV. For instance, the median age of sexual debut is 17.7 years, approximately 1 year prior to marriage, and the prevalence of sexual initiation among 15-19 year olds is $27 \%$. Further, of those who are currently engaging in premarital sexual activity, only $36 \%$ reported using a condom at last sex. The low rate of consistent condom use implies exposure to STIs, with $4 \%$ of adolescents 15-19 reporting having an STI or symptoms of a sexually transmitted disease and 5\% having acquired HIV. The prevalence of HIV more than doubles by the time girls reach the ages of 20-24 years [1].

The literature indicates that women and adolescent girls in many less developed settings are lacking the needed assets and capacities required to make more positive and healthier transitions that would facilitate their breaking out of persistent poverty and closing the economic and livelihoods gap between men and women. In such settings, women and girls' opportunities are inhibited by traditional practices, adverse gender norms and roles, and weak institutions and laws $[4,5]$. For instance, Duflo argues that inequality, poverty and the lack of access to economic assets, opportunities and labour markets are primary drivers for the persistent disadvantage of women relative to men [5] Other studies have directly linked gender power inequality to HIV risk behaviours and exposures [6]. Women and girls who have limited social capital and are more isolated engaged in riskier sexual behaviours, are more likely exposed to sexual violence and less likely to be tested for $\operatorname{HIV}[7,8]$.

To address these disadvantages, the Population Council, working in collaboration with the YWCA, Making Cents International, the National Savings and Credit Bank of Zambia (Natsave) and the Zambian Ministry of Health designed AGEP, a multi-sectoral and girlcentered intervention. The theory of change underlying AGEP (Fig. 1) proposed that working directly with adolescent girls to build their economic, health and social assets would facilitate positive change across a broad set of critical adolescent girls' experiences and outcomes, including early marriage and first birth, schooling attainment, age of sexual debut, sexual risk behaviour, and transmission of sexually transmitted infections, including HIV. The theory underlying AGEP defines assets as a store of value that can be drawn upon by adolescent girls to address challenges and overcome vulnerabilities [9-12]. It was posited that an asset-building framework operates by enhancing girls' knowledge and capacities, building confidence and efficacy, strengthening social networks, changing aspirations, and providing access to both health and financial services and resources. Further, it was theorized that such changes at the individual-level would percolate up to changes in the contextual environment within the family and community.

\section{Intervention design}

The goal of AGEP was to reach a minimum of 10,000 adolescent girls ages 10-19 across ten sites in four of the ten provinces of Zambia; half of the sites were urban areas and half rural areas. Provinces were purposefully selected in collaboration with the donor, weighing feasibility, resources for the programme and evaluation, and the objective of representing vulnerable adolescent girls. As the programme was specifically designed to reach the most-vulnerable adolescent girls, high-density urban housing compounds were targeted, while a more general sample of rural residential communities were targeted.

\section{Girls group meetings}

The AGEP intervention operated for a period of two years in each site from late 2013 and early 2014 through late 2015 and early 2016. Implementation of the programme was initiated sequentially by site, after the 


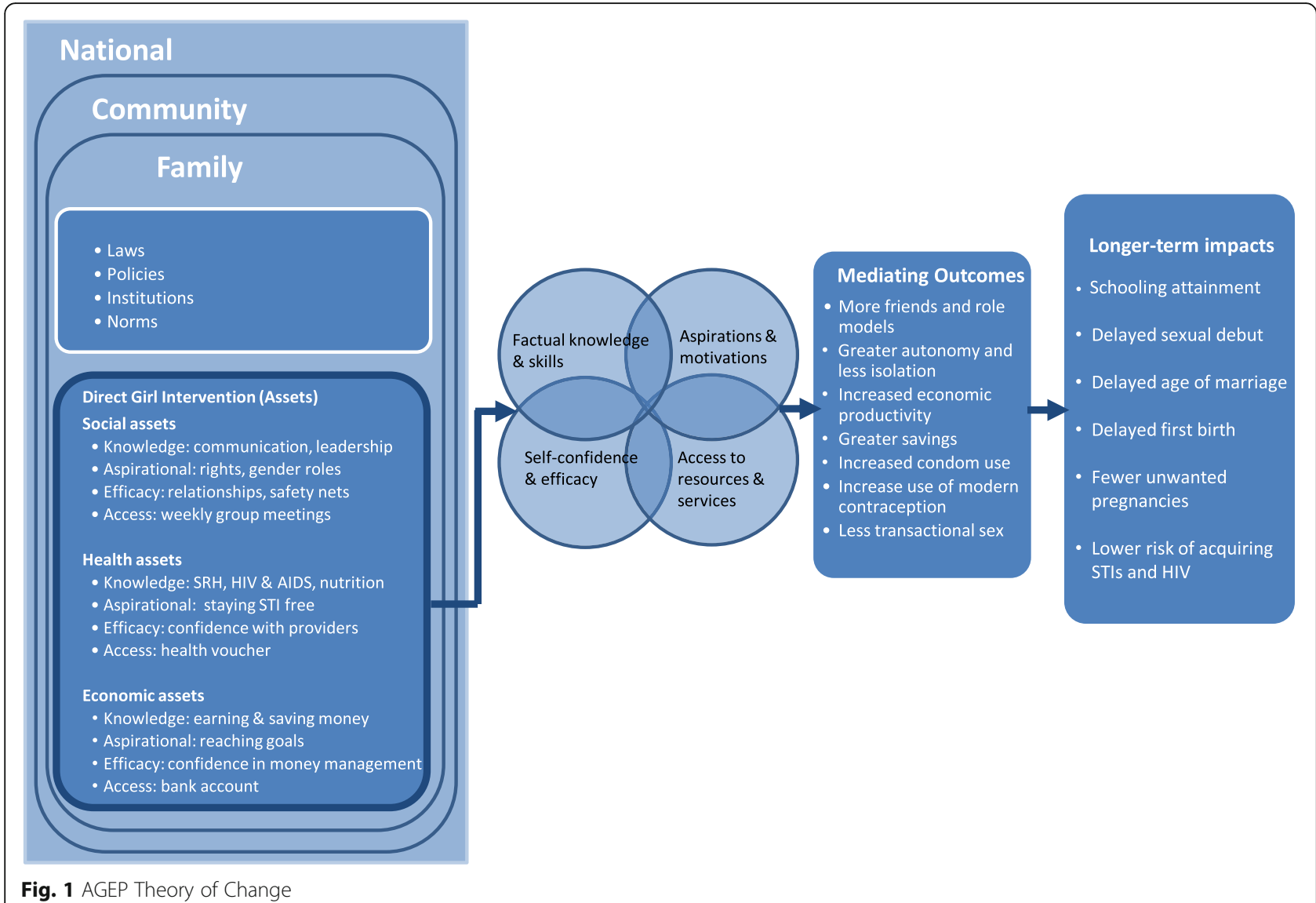

baseline evaluation survey was completed in each site. The core component of AGEP was group meetings in which 20 to 30 girls met at a local community space for one to two hours per week, most often on weekends. A community-based model was selected as school-based sexual reproductive health programs have been known to have limited impact because teachers find it hard to break out of their typical teaching styles and programs are adversely affected by an already low quality of schooling [13, 14]. In addition, school-based programs exclude out-of-school girls, which were meant to be included in the intervention. The girls' groups were stratified by age (10-14 and 15-19) and by marital/fertility status; girls stayed with their original groups as they aged or otherwise changed status. The group sessions were based on three core curricula (Table 1) and used illustrative vignettes, role play and participatory methods to maximize impact. The meetings were led by an older, young woman from the community who had been trained to initiate and guide educational sessions and exercises on a variety of subjects. The mentors were trained in groups at central locations at the beginning of the programme, with one refresher training approximately one year into the program. Monthly mentor meetings also took place with site coordinators to address mentor questions, experiences and provide short reviews of curriculum topics.

All the girls who were assigned to the intervention were invited to participate in AGEP girls' groups. Two additional programme components were randomly assigned to some of the girls groups. The additional components were: 1) the provision of a health voucher and, 2) an offer to open a bank savings account. Eligibility for these add-on components was determined by whether or not the girl group was located in a cluster that was randomized to receive the additional components of the intervention. The assignment of girls to the programme component is discussed further in the evaluation design section below.

\section{Health vouchers}

Girls who attended AGEP in randomly-selected areas to receive a health voucher targeted to improve access to ten general health and sexual and reproductive health services at partner public and private health providers (Table 2). The design and implementation of the AGEP health voucher was done in consultation with the Ministry of Health at the national, provincial and district level [15]. A published review of the literature found that health vouchers had been successful at increasing service 
Table 1 AGEP core curricula implemented through weekly girls' groups meetings

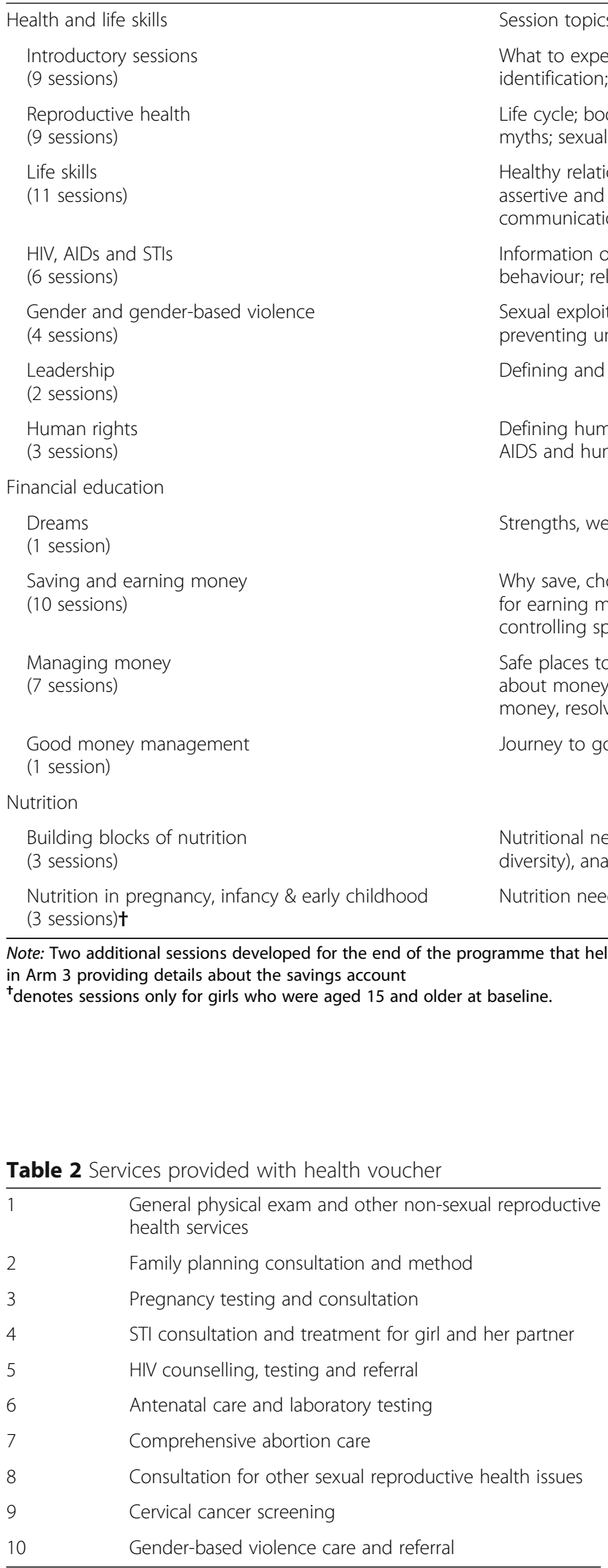

Note: Availability of services varied somewhat as not all facilities were able to provide the full list of services utilization and improving the quality of health services provision across a variety of health care and developing country settings. The review, however, noted that the breadth and scientific quality of evidence was limited and more rigorous assessments of effectiveness were needed [16]. A study of a programme that provided health vouchers specifically to adolescent girls in Nicaragua found that it directly reduced many of the barriers to adolescent sexual reproductive health care, including improving provider knowledge around adolescent health issues, attitudes and communication practices [17]. These studies suggested the potential for improving SRH care among adolescents in Zambia and the value of implementing a voucher programme embedded within a rigorous evaluation design.

In addition to the sexual and reproductive, nutritional and general health knowledge building during the weekly girls' groups, two additional programme elements supported the AGEP health voucher component: 1) the 
training of health facility staff in providing adolescent girl-friendly health services, and 2) a results-based financing of service provision at health facilities [18]. All participating health facility providers and staff were given a two-day training on adolescent girl-friendly health services that followed a set curriculum specifically designed for AGEP and supported by the Ministry of Health. Monthly monitoring and quality assurance visits to health facilities were also conducted [19]. The training sessions provided an overview of AGEP and the mechanisms of the health voucher, but also covered topics geared to improve the quality of service delivery for adolescents, including values clarification about family planning, HIV and STIs, communicating and counselling adolescents, identifying and addressing barriers to adolescent health care and the sexual and reproductive health rights of adolescents.

The results-based financing component of the health voucher also provided a basis from which to reimburse the health facility and provider for service use among adolescent girls. At private and non-governmental organization facilities, AGEP reimbursements were based on negotiated fees for each service provided. At public facilities, as health services are generally free, the payments to the facilities for each service, whose rates were agreed on with $\mathrm{MoH}$ and were uniform for all public facilities, were distributed by fixed percentages: to the providers $(50 \%)$, to the health facilities for supplies (25\%), for per-diems to the district health staff to assist in monitoring service provision (20\%), and to the District Health Office for overall management of the programme $(5 \%)$. Reimbursements were a function of the total number of girls with vouchers obtaining services and the number of services that they accessed during the period that the vouchers were usable. It should be noted that unlike other programme elements, due to delays in finalizing the arrangements, the health voucher started after one year of the programme and continued one year after the AGEP girls groups ended.

\section{Savings accounts}

While the girls groups provided capacity building in money management, budgeting and savings, the provision of a bank account was designed to provide a mechanism for knowledge and skills to be operationalized in practice. The bank account, provided to girls who would not otherwise typically have access to financial services, was hypothesized to reinforce girls' money management skills, promote economic asset building, grow a culture of savings, facilitate economic independence and provide assets in cases of emergencies or other basic needs. Savings accounts, as opposed to microcredit, were used as studies had shown in other settings limited results for such programs [20] and that micro- credit was not an appropriate first exposure to formal financial services for vulnerable adolescents [21]. Savings accounts, when appropriately designed for adolescents, had been shown in other contexts to improve financial literacy, increase the self-efficacy and savings behaviours [22]. Even in resource poor environments among vulnerable populations, savings accounts were found to increase savings and improve positive opinions about HIV prevention methods [23].

The bank account component was implemented in partnership with the National Savings and Credit Bank of Zambia (NatSave). The AGEP "Girls Dream" savings account provided adolescent girls a formal place to store money. The girls who were eligible for the savings account were provided an orientation in the girls' group sessions and a field trip was organized to the nearest NatSave branch to provide additional information and to open accounts. Girls who were under the age of 18 years were required to have co-signatories present at the account opening. The bank account was specifically tailored to the financial needs of the girls, with a very low minimum opening balance of KW 2.5 (US \$0.50) and the ability to deposit or withdraw funds with no fees. The girls who opened accounts could deposit on their own, although for girls under age 18 years the accounts required their co-signatories to withdraw funds. While no direct financial resources in the form of grants or loans were provided to AGEP girls, market research based on focus group discussions conducted during the pilot period suggested that adolescent girls had a variety of sources of income that may be used to build their savings, including cash from parents, paid piecemeal work, agricultural production, selling goods made and transactional sex [24].

\section{Intervention population}

As noted previously, AGEP was designed to serve a minimum of 10,000 vulnerable adolescent girls in Zambia aged 10 to 19 in ten sites located within four of the ten provinces of Zambia. The study provinces and the number of sites per province were selected purposefully through discussions with the donor regarding representation of the target population and consideration of the feasibility of operating AGEP while also conducting a randomized evaluation. Study sites within provinces were selected randomly from a sampling frame of potential sites, anchored to proximally-located health and banking facilities. The sampling frames of potential sites for urban and rural areas were developed separately. For urban areas, a list of high-density residential compounds was created, and sites selected randomly from the list, while in rural areas sites were randomly selected among areas that met the following conditions: that they were located within $15 \mathrm{~km}$ from a 
health facility and within $65 \mathrm{~km}$ of a NatSave bank branch.

AGEP was also designed to draw participants who expressed multiple levels of vulnerability through their residential location and the socio-economic characteristics of their households. To avoid over-representing girls in AGEP who have already experienced the outcomes that were to be prevented by the programme (early marriage, becoming pregnant, dropping out of school), these indicators were not directly used to identify vulnerability. Instead, an indicator of vulnerability was created using whether girls were behind in school for their age as a proxy, which also permits characterization of the vulnerability of girls younger than those who already have been married, pregnant or dropped out of school. Conceptually, the measure captures adolescents who are at risk of adverse outcomes, given the primacy of education in a child's life at these ages. Early in the schoolgoing process, many children fall behind in school due to late entry, repetition of grades, and temporary withdrawal from school; all of these events are a reflection of some degree of personal and household vulnerability. The information used to develop the measure of vulnerability was collected along with other household socioeconomic characteristics via a household survey in all study clusters, which was subsequently used as a sampling frame to determine eligible girls for participation in the programme with the most vulnerable girls selected from the list to receive invitations to participate.

\section{Evaluation methods and design}

The objective of the AGEP evaluation was to implement a rigorous randomized design to more confidently attribute the changes in girls' lives to their participation in AGEP. It was expected that the programme would have a positive impact on the longer-term demographic, reproductive and health outcomes of participating adolescents. It was also expected that the impact of the programme on these longer-term outcomes would be mediated through a set of individual assets or skills that the girls acquire through the programme. Given that the AGEP evaluation has multiple components that may lead to improved outcomes, the study was designed and statistically powered to evaluate the impact of each of these components on adolescent girl outcomes, independently for rural and urban areas.

\section{Randomised cluster design}

The AGEP evaluation was designed as a multi-arm randomised cluster trial where clusters within the ten study sites were randomised to receive different combinations (arms) of AGEP. The experimental and control arms of the study are displayed in Table 3, along with their associated components. Clusters assigned to the first
Table 3 Randomisation arms of AGEP

\begin{tabular}{ll}
\hline Study arm & AGEP components \\
\hline Intervention arm 1 & Weekly girls group mentor-led sessions \\
Intervention arm 2 & $\begin{array}{l}\text { Weekly girls group mentor-led } \\
\text { sessions + health voucher }\end{array}$ \\
Intervention arm 3 & $\begin{array}{l}\text { Weekly girls group mentor-led sessions + health } \\
\text { voucher + bank account }\end{array}$ \\
Control & No components of the AGEP intervention \\
\hline
\end{tabular}

intervention arm were assigned only the weekly girls' group sessions. Clusters in the second intervention arm were assigned the health voucher in addition to the weekly girls' group sessions, while clusters in the final intervention arm were assigned all components, including the bank account. The multi-arm evaluation provided for a rigorous assessment of the marginal benefit, as well as the incremental cost-effectiveness of each additional component of the intervention. All girls selected for participation in AGEP within a cluster were assigned to the intervention that was randomly selected for that cluster; girls in clusters for the control arm received neither interventions nor placebo exposures.

A cluster in the AGEP evaluation was delineated by Census Supervisory Areas (CSAs) that were compiled by the Zambia Central Statistical Office (CSO) for the 2010 national census. CSAs contained approximately 750 households in urban areas and 300 households in rural areas, although the average number of households varies considerably as population densities vary geographically. CSAs in urban areas were spatially relatively small, perhaps a few hundred metres long and wide, while CSAs in rural areas encompassed multiple kilometres. The assignment of CSAs to the experimental and control arms was conducted through a random selection process at a public lottery. The public lottery was conducted to maximize the transparency and community acceptance of AGEP. Local political and community leaders were invited to participate in the lottery, conducted at a centrally-located public facility. The lottery was conducted via a two-step selection process in which a CSA was first randomly selected for participation among all CSAs in the site and then an AGEP arm assigned through a second random selection process.

\section{Study outcomes}

The overall objective of AGEP was to make a meaningful change in the traditionally observed trajectories of vulnerable adolescent girls in Zambia as they pertained to early family formation, low schooling attainment and poor sexual and reproductive health. The process of change was posited to advance in phases, with the more immediate changes in the girls' assets and empowerment deriving from their participation in AGEP, translating into positive 
behavioural change and, ultimately, to longer-term change over the course of four years in which empowered adolescents would realize more positive transitional outcomes. As the concept of empowerment, mediating and longerterm outcomes are potentially broad and multifaceted, a set of representative indicators was used to epitomise their operationalization in the evaluation as noted in Table 4 . The indicators of empowerment ranged across the broad domains of social, economic and health and included measures of self-efficacy, social connections, positive gender normative beliefs, financial literacy, access to savings and sexual and reproductive health knowledge, among others. The longer-term indicators of programme impact ranged across three domains, socio-demographic, educational and health.

\section{Hypotheses}

The study will test several hypothesis to assess the impact of AGEP and its components. The hypothesis tests reflect the causal pathways as illustrated in the theory of change (Fig. 1). The impact of the intervention is posited to have operated through four general and domainspecific mechanisms, 1) by improving factual knowledge and building skills, 2) increasing self-confidence and efficacy, 3) increasing adolescent aspirations and motivations and, 4) improving access to key resources and services. The first set of hypotheses reflect the expectation that AGEP is having a strong and positive effect on the array of assets that girls can build upon and utilize to affect change. These are measured as immediate programme outcomes indicators in Table 4. A second set of hypotheses is made regarding the impact of the intervention on what are conceptualized here as mediating factors, reflected through HIV and pregnancy risk reduction behaviours, improved attendance in school and possessing greater economic resources and opportunities. The indicators used to reflect these hypotheses are presented as mediating outcomes indicators in Table 4. Finally, the overall objective and ultimate goal, of the programme is to improve the wellbeing of adolescent girls, reflected in specific ways, by reducing early marriage, early pregnancy and births, increasing educational attainment and reducing the acquisition of HIV and the Herpes Simplex Virus Type - 2 (HSV-2).

\section{Weekly girls groups meetings}

As noted above, all three intervention arms included weekly meetings in which girls met with a mentor and

Table 4 Primary and secondary study outcome indicators and measures

\begin{tabular}{|c|c|}
\hline Immediate programme outcomes & Asset domain \\
\hline Percentage of girls with high self-efficacy & Social \\
\hline Percentage of girls with strong role models and social support & Social \\
\hline Average number of friends, friends who can be counted on & Social \\
\hline Percentage of girls who hold positive gender normative believes & Social \\
\hline Average score on financial literacy scale & Economic \\
\hline Percentage of girls who have opened a formal bank account & Economic \\
\hline Average score on knowledge of sexual reproductive health & Health \\
\hline Average number of modern contraceptives known & Health \\
\hline Percentage having comprehensive knowledge of HIV & Health \\
\hline Percentage of girls accessing sexual and reproductive health services & Health \\
\hline \multicolumn{2}{|l|}{ Mediating outcomes indicators } \\
\hline Percentage of girls working for cash or in-kind in the past year & Economic \\
\hline Percentage of girls with a modest amount of savings & Economic \\
\hline Percentage of girls who have used a condom during last sex with a non-marital/non-cohabiting partner & Sexual and reproductive health \\
\hline Percentage of girls who are using modern contraception & Sexual and reproductive health \\
\hline Percentage of girls who have engaged in transactional sex & Sexual and reproductive health \\
\hline Longer-term outcomes indicators & Outcome domain \\
\hline Percentage of girls ever married & Socio-demographic \\
\hline Percentage of girls ever pregnant and given birth & Socio-demographic \\
\hline Percentage of girls experiencing unwanted pregnancy & Socio-demographic \\
\hline Percentage of girls completing grade 7 and grade 9 & Educational \\
\hline HIV prevalence among girls & Sexual and reproductive health \\
\hline Herpes Simplex Virus Type-2 (HSV-2) prevalence among girls & Sexual and reproductive health \\
\hline
\end{tabular}


each other to engage with a curriculum topic for the week, generally discuss their experiences and to socialize with each other. Given the randomized design, it is possible to assess the impact of the girls groups alone. The weekly girls' group meeting is hypothesized, in particular, to have positive effects on the girls' sexual and reproductive health, financial and nutritional knowledge, as well as build their communications, money management and self-advocacy skills. The group meetings also targeted the girls' social assets, including the number of friends reported, the quality of those relationships and whether they report a role model in their lives. Participation in the groups is also hypothesized to reduce social isolation and provide access to community spaces. These relationships and opportunities are, in-turn, hypothesized to weaken regressive gender norms and roles, move aspirations, and improve mediating behaviours, such as attending school. In the domain of schooling attainment, these changes are hypothesized to improve school participation, performance and, ultimately, attainment. If confirmed, these hypothesizes will result in strong and positive relationships between the indicator of randomization to the girls group only study arm and the indicators noted along this continuum of outcomes.

\section{Health voucher}

As with the bank account component, the health voucher intervention is hypothesized to directly increase access to health facilities and resources, as well as to increase efficacy and decision-making control over the utilization of health facilities and resources. The effect of the heath voucher component is expected to be distinguishable empirically over and above what is realized through the weekly girls groups alone. In the immediate term, the health voucher is hypothesized to increase the use of sexual and reproductive health services, including the use of modern contraception. Improved sexual and reproductive health, e.g., by obtaining counselling, care and treatment for STIs, is hypothesized to reduce HIV acquisition in the longer-term, while access to contraception is hypothesized to reduce unwanted pregnancy and birth outcomes. In general, it is hypothesized that as specific health issues are prevented or resolved, girls will have more positive assessment of their overall health.

\section{Bank accounts}

As noted in Table 1 above, the weekly girls' group curriculum includes 19 sessions on setting financial goals, budgeting and managing savings and expenditures. While the lessons themselves are hypothesized to directly increase knowledge and improve financial and entrepreneurial aspirations, the bank account component of AGEP was designed to provide a direct link and access to financial services to catalyse change. It is hypothesized, therefore, that the provision of a bank account will have positive impact on informal and formal savings behaviour. Increased access and control over economic resources is hypothesized to reduce the need for adolescent girls to rely on household resources from which they have less control to obtain personal items such as clothing and hygiene products, schooling supplies and/or pay school fees. Access to bank accounts is also hypothesized to depress the need for adolescent girls to rely on older and potentially riskier partners and exchange-based sexual relationships. These changes are hypothesized to reduce the prevalence of sexual exchange and, ultimately, reduce girls' acquisition of sexually transmitted infections, including HIV and HSV-2.

\section{Research population and sample sizes}

Participants for the research study were randomly selected among all invited AGEP participants who were never married at baseline, stratified by site and age group. For comparability, selection of the control girls followed a parallel process for identifying and selecting vulnerable girls, specifically a ranking of girls by their vulnerability score and randomly selecting girls below a set threshold, with the threshold determined by the total number of girls needed for recruitment into the programme at each site. As the ultimate objective of the funder and the programme was to improve the wellbeing of adolescent girls, the longer-term outcomes in Table 4 served as the focal indicators for the sample size calculations. As limited evidence was available for a sample size determination based on known effect sizes, minimally detectable effects were estimated using Optimal Design Plus Software Version 3.0 for a multi-site randomized trial to determine the most efficient sample size (number of clusters and respondents per cluster) given the budget available for fieldwork [25]. Sample sizes were estimated for the evaluation after four years, with conservative estimates of attrition, $20 \%$ for the younger cohort and 35\% for the older cohort. The samples were stratified by the younger and older cohorts (10-14 and 15-19) and urban and rural. The age stratification was necessary as there was no age mixing of groups in the weekly girls groups and certain aspects of the curricula were adjusted for age-appropriateness by cohort.

The minimally detectable effect sizes were estimated for comparison of each programme component against the control; estimates of effect sizes were not obtained for comparisons between the intervention arms themselves as little prior information was available to guide such an estimation approach. Given a total of ten study sites, it was determined that a total of 40 clusters per arm (four clusters per arm per site) and 20 participants per cluster was the most efficient combination. Baseline 
(control) estimates of study indicators by age group and residential status were obtained from the 2007 Zambia Demographic and Health Survey [26]. Statistical power of 0.80 and an alpha coefficient of 0.05 were set, while the effect size variability was fixed at 0.00 , as analyses would control for site fixed effects [25]. Table 5 provides estimates of prevalence in the control sample and minimal detectable effect sizes, by outcome and study stratifications.

The sample size calculations indicated that 3200 girls would be needed at the end of the study to assess all key study impact indicators: 800 girls (within 40 study clusters) in each of the three AGEP arms and in the control arm. An additional 400 girls (within 20 clusters) were designated to be sampled for urban areas in nearby high-density components. These girls and clusters were designed to serve as external controls given the potential for contamination between AGEP and control girls within the same site. Never married girls were targeted at baseline as a significant proportion of older girls would have already experienced the key outcomes that were meant to be impacted by the programme. To achieve the target sample of 3600 girls at the end of the study, the sample size was inflated for non-eligibility and non-response at baseline, attrition from the cohort and refusals for biological specimen collection. Thus, the final estimated sample to be collected at baseline was 7200 or 4800 in AGEP clusters, 1600 in internal control clusters and 800 in external control areas.

\section{Research instruments}

A household survey instrument was implemented prior to baseline to determine eligibility for the intervention and for selecting cases in designated control clusters. The household instrument included a roster of all household members with basic socio-demographic information on each member, household asset ownership, housing quality, recent household shocks, access to financial resources and distances to key resources, including schools, roads, health facilities, banks, etc. Comprehensive annual surveys were conducted at baseline and for four subsequent years. The baseline survey is included as an Additional file 1. The surveys instruments measure changes in attitudes, behaviour, transition status, social assets and cognitive skills that may occur over time regarding: educational attainment and schooling transitions; sexual activity, relationship status and sexual partners; marriage and marital dissolution; sexual and physical coercion and violence; gender attitudes, self- efficacy and locus of control; labour force participation and savings behaviour; living arrangements and household resources; mobility and migration; literacy, numeracy and cognitive skills; and ability; financial literacy and knowledge. Surveys conducted after the baseline also include questions of exposure to the intervention to address potential spillover impacts. All questionnaires were translated into Nyanja, Bemba and Kaonde.

Survey instruments were implemented by electronic data capture. Computer-Assisted Personal-Interviewing (CAPI) was used for questions that are non-sensitive. CAPI is a process of data capture in which the interviewer reads the question from a computer screen and enters the participant's response directly into a handheld or tablet device. For sensitive questions, including sexual behaviour, sexual violence, HIV risk perception, unwanted pregnancy and abortion, Audio Computer-Assisted Self-Interviewing (ACASI) was used. With ACASI the respondent listened with headphones to pre-recorded questions and response categories, while, if desired and if the participant was literate, simultaneously reading the question on the device screen. The participant entered a response by touching a designated number or option. ACASI maximizes confidentiality and privacy of response, as no one could hear or

Table 5 Estimates of prevalence in the control and minimally detectable effect sizes in percentage points (pp) of programme impact by age-cohort and by rural and urban stratifications

\begin{tabular}{|c|c|c|c|c|c|c|}
\hline & \multicolumn{2}{|l|}{ Total $^{\mathrm{a}}$} & \multicolumn{2}{|l|}{ Rural $^{b}$} & \multicolumn{2}{|l|}{ Urban $^{\mathrm{b}}$} \\
\hline & Younger & Older & Younger & Older & Younger & Older \\
\hline Ever had sex & $40 \%, \pm 10 \mathrm{pp}$ & $82 \%, \pm 9 \mathrm{pp}$ & $47 \%, \pm 14 \mathrm{pp}$ & $89 \%, \pm 11 \mathrm{pp}$ & $34 \%, \pm 13 \mathrm{pp}$ & $75 \%, \pm 13 \mathrm{pp}$ \\
\hline Ever married & $15 \%, \pm 6 \mathrm{pp}$ & $63 \%, \pm 10 \mathrm{pp}$ & $19 \%, \pm 11 \mathrm{pp}$ & $76 \%, \pm 13 p p$ & $10 \%, \pm 8 \mathrm{pp}$ & $48 \%, \pm 14 \mathrm{pp}$ \\
\hline Ever given birth & $16 \%, \pm 6 \mathrm{pp}$ & $70 \%, \pm 10 \mathrm{pp}$ & $18 \%, \pm 10 \mathrm{pp}$ & $83 \%, \pm 13 \mathrm{pp}$ & $13 \%, \pm 8 \mathrm{pp}$ & $54 \%, \pm 14 \mathrm{pp}$ \\
\hline Completed grade 7 & $61 \%, \pm 10 \mathrm{pp}$ & $60 \%, \pm 9 \mathrm{pp}$ & $43 \%, \pm 14 \mathrm{pp}$ & $41 \%, \pm 15 \mathrm{pp}$ & $79 \%, \pm 11 \mathrm{pp}$ & $85 \%, \pm 9 \mathrm{pp}$ \\
\hline Completed grade 9 & $28 \%, \pm 10 \mathrm{pp}$ & $38 \%, \pm 10 \mathrm{pp}$ & $11 \%, \pm 11 \mathrm{pp}$ & $17 \%, \pm 13 \mathrm{pp}$ & $45 \%, \pm 15 \mathrm{pp}$ & $64 \%, \pm 14 \mathrm{pp}$ \\
\hline Ever use of modern contraception & $15 \%, \pm 8 p p$ & $55 \%, \pm 7 \mathrm{pp}$ & $15 \%, \pm 12 \mathrm{pp}$ & $55 \%, \pm 14 \mathrm{pp}$ & $15 \%, \pm 12 \mathrm{pp}$ & $55 \%, \pm 15 \mathrm{pp}$ \\
\hline HIV prevalence & $6 \%, \pm 4 \mathrm{pp}$ & $12 \%, \pm 6 \mathrm{pp}$ & $6 \%, n / a^{c}$ & $7 \%, \mathrm{n} / \mathrm{a}^{\mathrm{c}}$ & $6 \%, n / a^{c}$ & $19 \%, \pm 10 \mathrm{pp}$ \\
\hline HSV-2 prevalence & $21 \%, \pm 8 \mathrm{pp}$ & $26 \%, \pm 8 p p$ & $21 \%, \pm 11 p p$ & $26 \%, \pm 12 p p$ & $21 \%, \pm 11 \mathrm{pp}$ & $26 \%, \pm 12 p p$ \\
\hline
\end{tabular}

Notes: Younger cohort members are expected to be ages 14-18 at endline; older cohort members are expected to be ages 19-23 at endline. All estimates assume alpha of 0.05 , power of 0.80 , and site fixed effects

${ }^{a}$ For each age cohort estimates are based on: 10 sites, 4 clusters per arm per site (40 clusters per arm), and 10 subjects per cluster

${ }^{b}$ For each age cohort estimates are based on: 5 sites, 4 clusters per arm per site (20 clusters per arm), and 10 subjects per cluster

${ }^{c}$ Not powered at 0.80 to detect programme impact 
see the question being read, nor the response option selected.

Biological markers were collected from adolescents aged 15 and older at baseline (Round 1), after Round 3 (at the end of AGEP), and Round 5 (at the end of the evaluation). HIV status was determined via capillary blood draws obtained from finger pricks. HIV tests were conducted by trained and certified voluntary counselling and testing (VCT) staff. In accordance with Ministry of Health guidelines, we conducted serial testing using Determine $^{\mathrm{Tm}}$ and Uni-Gold ${ }^{\mathrm{mx}}$ [27]. Both tests have a very high sensitivity (100\%) and specificity $(>99 \%)$ in controlled clinic evaluations, including a controlled laboratory setting in rural Kenya [28]. The respondent was provided the test results. Post-test counseling was guided by National Guidelines for Testing and Counselling [29].

The HSV-2 biological specimens were collected via finger prick. A sample of whole blood was collected and stored in microtainers and laboratory tested. To conduct the laboratory test, serum was derived from the whole blood and the Kalon ELISA assay used, for which the sensitivity and specificity have been found to be high (92\% and 98\%) respectively in African populations when compared to Western Blot [30]. Indeterminate results were not retested since their prevalence was so low as to make it impractical. After specimen collection all participants were provided information about HSV-2 detection, symptoms, safe sex practices and treatment options. Respondents were provided vouchers with identification numbers to receive their test results at AGEP participating health centers. A validation of the HSV-2 laboratory testing procedures was conducted prior to testing of specimens for the main study to assure the quality of laboratory testing protocols.

\section{Economic evaluation}

The study also includes an economic evaluation to rigorously assess the cost of implementing AGEP, as well as to assess the incremental cost-effectiveness of the addon components (health voucher and savings account) relative to the girls group intervention alone.. Direct programme costs were collected from AGEP budgets and financial reports and included both start-up and programme delivery costs for the Population Council and its partners. Participant-specific out-of-pocket and indirect costs data for participation in AGEP and uptake of services were also obtained. The relevant costs for these analyses are the real resource costs of delivering the programme services, not including the costs related to project evaluation. A decision analytic model will be constructed to generate estimates of the incremental costs per negative health outcome averted and positive progress achieved on non-health indicators from participating in AGEP. Incremental cost-effectiveness ratios
(ICERS) comparing the add-ons to the girls group only intervention will be calculated to assess whether costeffectiveness varies by study arm.

\section{Analysis}

The primary objective of the evaluation is to assess the impact of AGEP on adolescents' intermediate empowerment and longer-term outcomes using the above-noted hypothesis as test criteria. Important as well, analysis will be conducted to understand the underlying processes by which change occurred in the key areas of interest. As a first approach for assessing the impact of AGEP on empowerment and longerterm outcomes, an intent-to-treat analysis (ITT) will be conducted using the original randomised assignment to study arm as the primary indicator of impact. The average treatment effect of the programme on the adolescents in clusters randomised to AGEP relative to girls in clusters randomised to the control will be empirically assessed. The analysis will estimate models that are both unadjusted and adjusted for baseline covariates; both approaches accounting for strata and intraclass correlations within clusters. To accommodate for any baseline differences between AGEP and control clusters that may exist in the outcomes, a difference-in-differences (DID) calculation will be made for each indicator. The mid-term assessment after Round 3 focused on the immediate and mediating indicators as primary outcomes, while in the final assessment after Round 5 the mediating and longerterm outcomes will be the empirical focus.

As girls were invited to participate in AGEP, many choose not to do so, intermittently missed the weekly sessions or left the programme before the programme was completed. Therefore a secondary analysis will be conducted that uses a measure of participation intensity as the indicator of impact rather than the study's random assignment. It is important to recognize the statistical estimation problems that arise from self-selection into AGEP, and the degree of participation in it, with the characteristics of girls predicting both their uptake of and exposure to the programme and key outcomes and behaviours of interest. A two-stage instrumental variables estimation approach will be used in which the first stage will predict programme participation, using the exogenous randomised assignment as the key instrumental variable, observed baseline covariates will also be included. The second stage will then use the predicted participation intensity from the first stage along with baseline covariates to test the relevant hypotheses. Appropriate tests, including F-tests on excluded instruments, the Wald F-statistic, and the Hansen statistic for over-identification will quantitatively assess the credibility of the selected instrumental variable. 


\section{Discussion}

AGEP was an intervention whose objective was to improve important adolescent transitions of girls in Zambia, with the expectation that improved outcomes for girls would lead to life-long improvements in the well-being of women and their children. The rigorous randomized evaluation and cohort study to evaluate AGEP outlined in this protocol paper was designed to address information gaps and the weaknesses recognized in the existing literature, including non-randomized study designs, a lack of information on vulnerable and disadvantaged populations, and short-term durations of assessments [31]. As noted in the literature, the benefits from finding out what works, under what conditions and how for adolescents in a setting such as Zambia is large, as demographically the "cohort of young people age 10-24 is the largest in history," while epidemiologically developing countries face multi-burdens from diseases and are "characterized by high levels of all types of adolescent health problems" [32].

AGEP was a multi-sectoral intervention built off of the core concept of mentor-led, weekly girls' group meetings whose primary objective was to build and empower girls by 1) enhancing their knowledge and skills, 2) building their self-confidence and efficacy, 3) changing their aspirations, and 4) providing access to services and resources. The AGEP programme is unique in the amount of investment in this effort, with over two years of weekly programme activities devoted to girls' empowerment across a range of domains, including sexual and reproductive health, financial literacy, life skills and nutrition. AGEP was not, however, limited to the weekly girls groups, as two additional components, a bank account and health voucher, enhanced the potential impact of the group learnings by providing girls direct access to resources that typically did not exist for them or have high barriers to use. The multi-arm randomized evaluation was designed to assess whether each of these programme components provided additional value added in terms of impact.

Determining the impact of AGEP is important in order to provide needed evidence for governments, donors, and other stakeholders to develop policies and programs for adolescent girls. Delineating costs of the programme and the investment needed for added programme components can provide additional critical information about the investment needed to scale the programme and the incremental costs of investments in each intervention component. To achieve this additional aim, the study research protocol included an embedded economic evaluation that collected programme implementation and participant costs. The economic evaluation will provide cost-effectiveness information for each study arm and an opportunity to compare AGEP with alternative programmes that address the needs of adolescent girls in the areas of early marriage and childbearing, education and sexual and reproductive health.

\section{Additional file}

Additional file 1: AGEP Baseline Survey. (XLSX 2922 kb)

\section{Abbreviations}

ACASI: Audio Computer-Assisted Self-Interviewing; AGEP: Adolescent Girls Empowerment Programme; CAPI: Computer Assisted Personal Interviewing; CSA: Census Supervisory Area; DFID: Department for International

Development; HIV: Human immunodeficiency virus; HSV-2: Herpes simplex virus, type-2; $\mathrm{MOH}$ : Ministry of Health; NATSAVE: National Savings and Credit Bank of Zambia; STI: Sexually transmitted infections; UNZA-REC: University of Zambia, Research Ethics Committee; YWCA: Young Women's Christian Association

\section{Acknowledgements}

AGEP is being implemented by a consortium led by the Population Council, the Young Women's Christian Association (YWCA), Making Cents International, the National Savings and Credit Bank of Zambia and the Ministry of Health, Government of the Republic Of Zambia. We thank the consortium members, as well as members of the AGEP Evidence Scrutiny Committee, DFID-Research and Evidence Division, and Mott MacDonald. The authors also thank the UK Department for International Development (DFID) for investing in rigorous evaluation and, in particular, Valerie Roberts who has been a champion of AGEP.

\section{Funding}

The AGEP programme and evaluation was funded by UK aid from the UK government; however the views expressed do not necessarily reflect the UK government's official policies.

\section{Availability of data and materials}

Materials related to this study can be found on the Population Council: http://www.popcouncil.org/research/adolescent-girls-empowermentprogram.

\section{Author's contributions}

$\mathrm{PCH}, \mathrm{KA}, \mathrm{ESH}, \mathrm{JB}$, and FB designed the study with review and suggestions from UKAID-DFID staff and an independent consulting company hired as an external reviewer. $\mathrm{PCH}, \mathrm{ESH}, \mathrm{FB}, \mathrm{KA}$ and $\mathrm{JB}$ drafted the study protocol and instruments. PCH drafted the manuscript, KA, ESH, JB, FB, NJ, MB reviewed the draft manuscripted, contributed text and provided and feedback. All authors read and approved the final manuscript.

\section{Competing interests}

The authors declare that they have no competing interests.

\section{Consent for publication}

Not applicable.

\section{Ethics approval and consent to participate}

The research protocol was approved by the Population Council Institutional Review Board (PCIRB - protocol \#581), which has a Department of Health and Human Services (DHHS) federal-wide assurance number of

FWA00000279. The Population Council IRB has established procedures that adhere to the U.S. Federal guidelines for human subjects as set forth in Title 45, Part 46 of the Code of Federal Regulations (Department of Health and Human Services 1991). The protocol was also reviewed by the University of Zambia's Research Ethics Committee (UNZA-REC - protocol 008-11-12) with federal-wide assurance number is FWA00000338. All study investigators and staff were certified in human subject's protection training prior to study initiation. All study participants provided written consent if they were age 18 and older; assent and guardian written informed consent was obtained for all participants under the age of 18. A two staged consent process was conducted, the first for the behavioural survey and the second for the biomarker data collection. 


\section{Author details}

'Population Council, 4301 Connecticut Avenue, Washington, D.C. 2008, USA. ${ }^{2}$ Population Council, P.O. Box 17643-00500, Nairobi, Kenya. ${ }^{3}$ Population Council, One Dag Hammarskjold Plaza, New York, NY 10017, USA.

${ }^{4}$ Independent consultant, 08019 Barcelona, Fluvia 101, 3o 4a, Spain. ${ }^{5}$ Departments of Economics and Sociology, University of Pennsylvania, 3718 Locust Walk, Philadelphia, P.A. 19104, USA. 'Eondon School of Hygiene and Tropical Medicine, 15-17 Tavistock Place, London WC1H 9SH, UK. PPopulation Council, Plot 3670, No. 4, Mwaleshi Road, Olympia Park, 10101 Lusaka, Zambia.

Received: 11 February 2017 Accepted: 21 April 2017

Published online: 05 May 2017

\section{References}

1. Central Statistical Office (CSO) MoHM, [Zambia], and ICF International: Zambia Demographic and Health Survey 2013-2014. In. Rockville, Maryland USA: Central Statistical Office, Ministry of Health and ICF international 2014.

2. National Research Council and Institutes of Medicine: Growing up global: The changing transitions to adulthood in developing countries. Washington D.C. U.S.A.: National Academies Press; 2005.

3. Central Statistical Office (CSO) MoHM, [Zambia], and ICF International: Zambia Demographic and Health Survey 2013-2014 [Dataset]. ZMIR61FL. DTA. In. Rockville, Maryland USA: Central Statistical Office, Ministry of Health and ICF international; 2014.

4. World Bank. World development report: gender equality and development. World Bank: Washington, D.C. U.S.A; 2012.

5. Duflo E. Women empowerment and ecnomic development. J Econ Lit. 2012;50(4):1051-79.

6. Pettifor AE, Measham DM, Rees HV, Padian NS. Sexual power and HIV risk. South Africa Emerg Infect Dis. 2004;10(11):1996-2004.

7. Hallman K. Gendered socioeconomic conditions and HIV risk behaviours among young people in South Africa. African Journal of AIDS Research. 2005;4(1):37-50.

8. Hallman K: Social exclusion: The gendering of adolescent HIV risks in KwaZulu-Natal, South Africa. In: The Fourth Wave: Violence, Gender, Culture \& HIV in the 21st Century. edn. Edited by Klot JF, Nguyen V-K. Paris, France: UNESCO; 2011.

9. Bruce J, Sebstad J: Building assets for safe and productive lives: A report on a workshop on adolescent girls' livelihoods, Adolescent girls livelihoods meeting: In. New York, NY: Population Council; 2004

10. Dickson K, Bangpan M. Providing access to economic assets for girls and young women in low-and-lower middleincome countries. A systematic review of the evidence. London: EPPI-Centre, Social Science Research Unit, Institute of Education, University of London; 2012.

11. Austrian K, Ghati D. Girl centered program design: a toolkit to develop strengthen \& expand adolescent girls programs. Population Council: New York, NY; 2010.

12. Ashburn $\mathrm{K}$, Warner A. Can economic empowerment reduce vulnerability of girls and young women to HIV? Emerging insights. International Center for Research on Women: Washington D.C., U.S.A; 2010

13. Plummer MJ, Wight D, Obasi A, Wamoyi J, Mshana G, Todd J, Mazige B, Makokha M, Hayes R, Ross D. A process evaluation of a school-based adolescent sexual health intervention in rural Tanzania: the MEMA kwa Vijana programme. Health Educ Res. 2007;22(4):500-12.

14. Amin S, Laila Rahman, Ainul S, Rob U, Zaman B, Akter R: Enhancing adolescent financial capabilities through financial education in Bangladesh. In. New York, NY U.S.A.: Population Council; 2010.

15. Gorter AC: Health Vouchers for Adolescent Girls Empowerment Program. In. Unpublished report: Independent consultant; 2012.

16. Bellows NM, Bellows B, Warren CE. The use of vouchers for reproductive health services in developing countries: systematic review. Trop Med Int Health. 2011;16(1):84-96.

17. Meuwissen LE: Can a comprehensive voucher programme prompt changes in doctors' knowledge,attitudes and practices related to sexual and reproductive health care for adolescents? A case study from Latin America. Trop Med Int Health 2006, 2(6):889-898.

18. World Bank: A guide to competative vouchers in health. In. Washington D. C.: The International Bank for Reconstruction and Development/The World Bank; 2005.
19. Population Council: Curriculum on adolescent-friendly health services and health voucher mechanisms. In. New York: Population Council; 2015.

20. USAID. Reducing adolescent Girls' vulnerability to HIV infection: examining microfinance and sustainable livelihoods approaches: a literature and program review. United States Agency for International Development: Washington D.C U.S.A; 2008.

21. Erulkar A, Chong E: Evaluation of a savings and micro-credit program for vulnerable young women in Nairobi. In. New York, N.Y. U.S.A: Population Council; 2005

22. Austrian K, Muthengi E. Safe and smart savings products for vulnerable adolescent girls in kenya and uganda: evaluation report. Population Council: Nairobi, Kenya; 2013

23. Ssewamala FM, Alicea S, Bannon WM Jr, Ismayilova L. A novel economic intervention to reduce HIV risks among school-going AIDS orphans in rural Uganda. J Adolesc Health. 2008;42(1):102-4

24. James-Wilson D, Mani R, Torres V: Adolescent Girls Empowerment Program Youth-Inclusive Market Research Report-Round One In. Unpublished report: Making Cents International; 2012

25. Spybrook J, Bloom H, Congdon R, Hill C, Martinez A, Raudenbush S: Optimal Design Plus Empirical Evidence: Documentation for the "Optimal Design" Software In.; 2011.

26. Central Statistical Office (CSO) MoHM, Tropical Diseases Research Centre (TDRC), University of Zambia, and Macro International Inc.: Zambia Demographic and Health Survey 2007. In. Calverton, Maryland: CSO and Macro International Inc.; 2009.

27. National HIV/AIDS/STI/TB Council: Zambia HIV Rapid Test Algorithm. In. Lusaka, Zambai: National HIV/AIDS/STI/TB Council; 2008.

28. Foglia GF, G. Donald Royster IV, Wasunna KM, Kibaya R, Malia JA, Calero EK, Sateren W, Renzullo PO, Robb ML, Birx DL et al. Use of rapid and conventional testing Technologies for Human Immunodeficiency Virus Type 1 serologic screening in a rural Kenyan reference laboratory. J Clin Microbiol. 2004(Aug 2004):3850-5852.

29. Ministry of Health GotRoZ: Zambia National Guidelines for HIV Counseling and Testing of Children. In. Lusaka, Zambia: Ministry of Health, Government of the Republic of Zambia; 2011.

30. van Dyck E, Buve A, Weiss HA, Glynn JR, Brown DW, De Deken B, Parry J, Hayes RJ. Performance of commercially available enzyme immunoassays for detection of antibodies against herpes simplex virus type 2 in African populations. J Clin Microbiol. 2004;42(7):2961-5.

31. Salam RA, Das JK, Lassi ZS, Bhutta ZA. Adolescent health interventions: conclusions, evidence gaps, and research priorities. J Adolesc Health. 2016:59(S4):S88-92.

32. Fatusi A. Editorial: young People's sexual and reproductive health interventions in developing countries: making the investments count. J Adolesc Health. 2016;59(3):S1-3.

\section{Submit your next manuscript to BioMed Central and we will help you at every step:}

- We accept pre-submission inquiries

- Our selector tool helps you to find the most relevant journal

- We provide round the clock customer support

- Convenient online submission

- Thorough peer review

- Inclusion in PubMed and all major indexing services

- Maximum visibility for your research

Submit your manuscript at www.biomedcentral.com/submit 